\title{
VV ORIONIS: A WELL-BEHAVED EARLY-TYPE ECLIPSING BINARY SYSTEM*
}

\author{
CARLSON R. CHAMBLISS
}

Kutztoun University, Kutztown, Pennsylvania, U.S.A.

(Received 27 July, 1983)

\begin{abstract}
VV Orionis is a detached eclipsing binary system consisting of stars of spectral types BI V and B4 V with a period of $1 .{ }^{\mathrm{d}} 4854$. There is also a third component whose orbital period about the eclipsing pair is about 120 days. In recent years there have been several new photoelectric and spectroscopic investigations of this system, and the results of these are compared. Both the geometric and photometric orbital elements appear to be well determined. VV Ori A appears to be of normal size and mass for its spectral class, but VV Ori B seems to be somewhat smaller than would be expected for a normal B4 V star. Linear limb-darkening coefficients are derived for VV Ori A, and these are in good agreement with theory. This system is of particular importance, because it is only one of very few earlytype systems for which reliable limb-darkening coefficients can be expected to be obtained. The contribution of the light of the third component to the system has also been determined, and it appears most likely that VV Ori $\mathrm{C}$ is a star of spectral type A3 V.
\end{abstract}

\section{Introduction}

VV Orionis (HD 1868) is a detached eclipsing binary system having a period of $1^{\mathrm{d}} .4854$. The eclipses are complete, the primary is a transit, and both components are Main-Sequence stars of spectral type B. The details of the earlier history of this system are summarized by Wood (1946). More recent photoelectric investigations include those made by Huffer and Kopal (1951), Bolokadze (1953), and by Atkins (1971). Orbital elements were calculated by each of these investigators, and Kopal and Shapley (1956) obtained an additional set of orbital elements based upon observations made by Huffer in blue and in yellow light.

The earliest radial velocity curve of VV Ori was obtained by Daniel (1916), who was also able to show that periodic variations in the residuals of the radial velocities could be interpreted as due to the presence of a third body in the system. He estimated the period of the third component to be about 120 days. Although the mass of this component has been approximately determined from spectroscopic analysis, its contribution to the light of the system has either been ignored or has been only crudely approximated in most photometric analyses of this system.

It has long been recognized that VV Ori should be capable of yielding reliable

* Paper presented at the Lembang-Bamberg IAU Colloquium No. 80 on 'Double Stars: Physical Properties and Generic Relations', held at Bandung, Indonesia, 3-7 June, 1983. 
limb-darkening coefficients (Koch et al., 1963). In fact, apart from SZ Cam, VV Ori is practically the only early-type eclipsing binary system for which highly reliable limb-darkening coefficients can presently be expected to be determined. Most photometric analyses of VV Ori, however, have concentrated on the geometrical elements of the system, and only assumed or approximate values of the limb darkening have generally been used or reported.

\section{Recent Investigations of VV Orionis}

Recent photometric investigations of VV Ori include those by Duerbeck (1975), Eaton (1975), and by Chambliss (cf. Chambliss and Leung, 1982). The observations of Duerbeck were obtained at the ESO in one season during 1973-74 in UBV and in $\mathrm{H} \beta$. The observations of Eaton are from the OAO-2 and are in eight bandpasses ranging from $1330 \AA$ to $4250 \AA$. The observations of Chambliss were obtained at KPNO in four seasons during 1975-79 in $U B V$ and in $u v b y$.

Several analyses have been published from these investigations. Duerbeck analyzed his $U B V$ data by means of the method of Russell and Merrill (1952) and the iterative procedure of Irwin (1947). Eaton analyzed five of his light curves with the use of the method of Wilson and Devinney (1971). A unique geometry was assumed for all five light curves. A similar approach was used by Leung in analyzing light curves formed from the normal points of Chambliss. All seven light curves were analyzed simultaneously, and a unique geometry was assumed.

Other investigations have also been made using these sets of data. The data of Duerbeck were analyzed by Budding and Najim (1980) using a frequency-domain analysis procedure. The independent treatment of $L_{1}$ and $L_{2}$ permitted the small contribution of the light of the third component to be taken into consideration. Cester et al. (1978) reanalyzed the $U B V$ light curves of Duerbeck and the $3320 \AA$ and $4250 \AA$ light curves using the model of Wood $(1971,1972)$. The most recent photometric analyses of VV Ori have been those by Giuricin et al. (1983) and by Chambliss (1983) using the Wood model on the observations of Chambliss.

As might be expected, the different methods of analysis used by different investigators on different bodies of data do not all produce the same results. As was noted by Giuricin et al. (1983) and by Chambliss (1983), the attempt by Leung to fit all seven light curves of Chambliss to a unique geometry resulted in a compromised solution. The derived inclination $\left(i=90^{\circ} 0\right)$ was plainly too high, as all other recent investigations of VVOri have yielded $i=85^{\circ}$ or $86^{\circ}$. Other criticisms of this solution are also given in the two later papers.

Only the Trieste astronomers have analyzed all three of these sets of photometric data using the same method: i.e., the Wood model. The agreement between the different sets of elements obtained by them is very good. In fact, the agreement between the orbital elements derived from the three independent sets of data but analyzed with the same method is much better than between the orbital elements 
reported by Chambliss and Leung and those reported by Giuricin et al., both of which used the normal points of Chambliss in their analysis. Thus it appears that the computed orbital elements of a well-observed eclipsing binary seem to depend more on the method of analysis chosen than they do on whose data base is utilized.

The formal errors quoted by Chambliss and by Giuricin et al. average about \pm 0.002 for the parameters $r_{1}$ and $k$. It appears that this is about the highest precision that one can expect to obtain for these parameters by using the Wood model. Although the errors for $r_{1}$ and $r_{2}$ quoted in Leung's solution, which made use of the Wilson-Devinney method, are lower than this, it does not seem likely that the derived parameters are reliable to the precision implied by these formal errors $( \pm 0.0004)$. It is interesting to note that the errors for $r_{1}$ and $k$ of VV Ori quoted in the solution tabulated in the paper by Kopal and Shapley (1956) are about \pm 0.003 . In this paper the iterative method of Kopal was used to solve the light curves, and it would appear that this method is capable of yielding nearly as satisfactory solutions for systems such as VV Ori as is the Wood model.

Of the various investigations only those by Kopal and Shapley, Duerbeck, Eaton, and Chambliss attempted to determine limb darkening coefficients empirically. Duerbeck reported values of $u=0.07,0.14$, and 0.17 for VV Ori A in $V$, $B$, and $U$, respectively. These values are much lower than the predicted theoretical values, but Duerbeck notes they may be the consequence of the asymmetries which he observed in the annular portion of the primary eclipse. The value of $0.5 \pm 0.1$ quoted by Kopal and Shapley for VV Ori A in blue light is higher than one would predict from theory. Eaton's values are more in line with the theoretical values for the limb darkening coefficients, but most of his bandpasses lie well outside the optical range. The results obtained by Chambliss are summarized in the next section.

Only the papers by Budding and Najim and by Chambliss attempted to empirically determine the contribution of the third component to the light of the system. It is the belief of this author that the values of $L_{3}$ which were obtained by Budding and Najim are too low.

There have been two recent spectroscopic investigations of VV Ori. These are by Beltrami and Galeotti (1970) and by Duerbeck (1975). The former investigation was made at a lower dispersion, but the masses reported for the components $\left(\mathfrak{M}_{1}=10.2 \mathfrak{M}_{\odot}, \mathfrak{M}_{2}=4.5 \mathfrak{M}_{\odot}\right)$ are realistic for stars of spectral types $\mathrm{B} 1 \mathrm{~V}$ and $\mathrm{B} 4 \mathrm{~V}$, respectively, while those reported by Duerbeck $\left(\mathfrak{M}_{1}=7.60\right.$ $\mathfrak{M}_{\odot}, \mathfrak{M}_{2}=3.42 \mathfrak{M}_{\odot}$ ) appear to be much too low.

\section{The Physical Nature of VV Orionis}

The photometric properties of VV Ori are summarized in the papers of Duerbeck and of Chambliss and Leung. In the latter paper the following magnitudes are 
given :

\begin{tabular}{lccc}
\hline & Maximum & Primary & Secondary \\
\hline$V$ & +5.33 & +5.66 & +5.50 \\
$B-V$ & -0.19 & -0.18 & -0.19 \\
$U-B$ & -0.92 & -0.90 & -0.93 \\
\hline
\end{tabular}

Chambliss estimates $E(B-V)$ as +0.07 implying that $(B-V)_{0}=-0.26$ and $(U-B)_{0}=-0.97$ for VV Ori. This star is a member of the I Orion association, and its distance is estimated to be about 400 parsecs.

All published times of minimum light observed for this system are listed in these two papers, and the period is given in both as $1^{\mathrm{d}} .485378$. All available data indicate that the period of VV Ori has remained constant for the past $70 \mathrm{y}$. The third component will introduce a small light-time effect - a total amplitude of approximately two minutes - on the times of minimum light, but it has not proven possible to extract the orbital period of VV Ori $\mathrm{C}$ from variations in the observed times of minimum light of the eclipsing pair.

The light curves of Chambliss do not show some of the peculiarities reported by Duerbeck for his light curves, and this author doubts that most of these effects are regularly present in the light curves of VV Ori. Most notable of these is the dip at about 0.65 phase present in all three of Duerbeck's light curves. In the light curves of Chambliss only the intermediate-band $u$ curve shows this effect and only to a much smaller extent than is the case with the light curves observed by Duerbeck. Duerbeck's model of two gas streams as a possible explanation for this effect raises questions as to how such streams could arise from both components of a dynamically detached system.

Unlike those of Duerbeck, the light curves of Chambliss are symmetrical in the annular phase about primary minimum. Were this not the case, reliable limb darkening coefficients for the primary component could not be obtained, and the low values for $u_{1}$ obtained by Duerbeck are probably attributable to the peculiarities which he observed at this phase of the light cycle.

The geometrical elements reported in the various analyses of VV Ori are in substantial agreement with each other. The following are taken from the 1983 paper of Chambliss :

\begin{tabular}{llll}
\hline$i$ & $85^{\circ} .60 \pm 0^{\circ} .17$ & $k$ & $0.4864 \pm 0.0025$ \\
$r_{1}$ & $0.3627 \pm 0.0017$ & $r_{2}$ & $0.1764 \pm 0.0014$ \\
$a_{1}$ & 0.3830 & $a_{2}$ & 0.1796 \\
$b_{1}$ & 0.3713 & $b_{2}$ & 0.1770 \\
$c_{1}$ & 0.3588 & $c_{2}$ & 0.1755 \\
\hline
\end{tabular}

The system is detached. The oblateness of the primary component is about $6 \%$, while that of the secondary is only about $2 \%$. 
One of the main reasons for studying VV Ori is to obtain reliable empirical limb darkening coefficients. As has already been noted, VV Ori is one of the very few early-type systems for which this is possible. It is really only possible to obtain limb darkening coefficients for the primary component, since the secondary component contributes less than $10 \%$ of the light of the system. Furthermore the portion of the light curves which would be most sensitive to the value of $u_{2}$ would be the secondary eclipse. This eclipse, however, is a complete occultation, and hence, observations made in the total portion of secondary minimum would contain no information at all about $u_{2}$. Listed below are theoretical values for the limb darkening $\left(u_{1}^{*}, u_{2}^{*}\right)$ taken from the data given by Al-Naimiy (1978) and the empirical values for $u_{1}$ adopted by Chambliss in his 1983 paper:

\begin{tabular}{llll}
\hline$\lambda$ & $u_{1}^{*}$ & $u_{1}$ & $u_{2}^{*}$ \\
\hline$V$ & 0.28 & $0.26 \pm 0.05$ & 0.32 \\
B & 0.35 & $0.32 \pm 0.04$ & 0.41 \\
$U$ & 0.33 & $0.35 \pm 0.05$ & 0.35 \\
$y$ & 0.28 & $0.29 \pm 0.03$ & 0.32 \\
$b$ & 0.34 & $0.25 \pm 0.04$ & 0.39 \\
$v$ & 0.37 & $0.29 \pm 0.05$ & 0.42 \\
$u$ & 0.34 & $0.27 \pm 0.04$ & 0.36 \\
\hline
\end{tabular}

The agreement between theory and observation in this case is remarkably good.

Attempts were also made by Chambliss to derive non-linear limb darkening coefficients from his VV Ori observations. The formula treated in the Wood model is that of Klinglesmith and Sobieski (1970), namely:

$$
I(u)=I_{0}(1-A(1-\mu)-B \mu \log \mu)
$$

where

$$
\mu=\cos \gamma
$$

All attempts to obtain reliable values for $A$ and $B$ proved unsuccessful, however, and this supports the contention of Grygar et al. (1972) who stated that non-linear limb-darkening laws are beyong the reach of the presently available observations of close eclipsing binary stars - even if these are well-behaved in most other respects.

Of the recent papers on VV Ori only two, those of Budding and of Najim (1980) and of Chambliss (1983), have attempted to empirically determine the light of the third component. The values of $L_{3}$ derived in the former are too low to be compatible with the existing spectroscopic data, but the latter indicates that the third component is most likely a star of spectral type A3 V. The fractional 
luminosities adopted by this investigator are as follows:

\begin{tabular}{llll}
\hline & $L_{1}$ & $L_{2}$ & $L_{3}$ \\
\hline$V$ & 0.891 & 0.092 & 0.017 \\
$B$ & 0.895 & 0.091 & 0.014 \\
$U$ & 0.925 & 0.070 & 0.005 \\
$y$ & 0.894 & 0.089 & 0.017 \\
$b$ & 0.899 & 0.086 & 0.015 \\
$v$ & 0.907 & 0.081 & 0.012 \\
$u$ & 0.929 & 0.066 & 0.005 \\
\hline
\end{tabular}

On the basis of the photometric data a temperature of $25000 \mathrm{~K}$ has been assigned to the primary component. In the Wood model the temperature of the secondary component is usually treated as an independent variable while that of the primary is held constant. A mean temperature of $15650 \pm 90 \mathrm{~K}$ for $T_{2}$ is derived. These temperatures are compatible with spectral types of B1 V and B4 V, respectively. Both Eaton and Leung used values of $21000 \mathrm{~K}$ for $T_{1}$ in their solutions. This value is not compatible, however, with the presently accepted temperature scales for stars having the spectroscopic and photometric properties of VV Ori A.

As has already been noted, there is still much controversy over the spectroscopic orbital elements of VVOri. Duerbeck describes VVOri as a double-lined spectroscopic binary, but this contention has been challenged by Andersen (1976), whose comments have in turn been questioned by Duerbeck (1976). VV Ori B contributes only $\sim 10 \%$ of the light of the system (somewhat less than this according to Chambliss, Leung, Giuricin, and Kopal and Shapley; somewhat more than this according to Duerbeck). Thus it is difficult to see how this component can display spectral features over almost all orbital phases. Furthermore the masses of the components as reported by Duerbeck are decidedly low. The values given by Beltrami and Galeotti (1970) imply that the two components are of fairly normal size and mass, but Duerbeck's spectroscopic orbital elements imply components which are severely undersized and undermassive for their luminosities. These two papers agree on the orbital period of the third components $\left(115^{\mathrm{d}} .9\right.$ and $119^{\mathrm{d}} .1$, respectively), but the former assigns a mass of $2.3 \mathfrak{M}_{\odot}$ to this component, while the latter estimates that VV Ori $\mathrm{C}$ has a mass of $1.6 \mathfrak{M}_{\odot}$. According to this investigator, the spectral type of this component is $\mathrm{A} 3 \mathrm{~V}$, and the normal mass for a star of this type is $2.1 \mathfrak{M R}_{\odot}$.

The following are believed to be the most realistic estimates for the absolute dimensions of the components of VV Ori:

\begin{tabular}{lcll}
\hline$R_{1}$ & $4.94 \odot$ & $R_{2}$ & $2.36 \odot$ \\
$\mathfrak{M}_{1}$ & $10.2 \odot$ & $\mathfrak{M}_{2}$ & $4.5 \odot$ \\
$L_{1}$ & $8425 \odot$ & $L_{2}$ & $260 \odot$ \\
$M_{\text {bol1 }}$ & -5.01 & $M_{\text {bol2 }}$ & -1.24 \\
\hline
\end{tabular}


The larger component appears to be normal for a star of spectral type B1 V and is found to be fairly close to the ZAMS. The secondary component, however, appears to be unusually small for its mass.

\section{Conclusions}

Although many of the problems concerning VV Ori appear to have been solved, a new radial velocity curve is definitely needed. Even if VV Ori does prove to be only a single-lined system, new values for $K_{1}$ and $f(\mathfrak{M})$ are still urgently needed.

The peculiarities of the light curve of VVOri discussed by Duerbeck have not been confirmed by Chambliss: and it seems that the light variations of this system can be described as being essentially without complications.

The limb darkening results for VV Ori A are particularly important, since they are the most precisely determined linear limb darkening coefficients which have been found thus far for an early-type star. They are essentially in good agreement with the values determined in recent theoretical studies, and this fact should inspire confidence in those researchers who use the results of such studies when deriving the other orbital elements of eclipsing binary systems. The failure of attempts to obtain reliable empirical non-linear limb darkening coefficients supports the view that non-linear limb darkening laws are beyond the reach of the presently available observations of eclipsing binary stars.

VV Ori is a detached system, and the components are sufficiently far apart for the close proximity effects to be relatively weak. Although VV Ori appears to be small for its mass, VV Ori A is an archetypal early B-type Main-Sequence star.

\section{References}

Al-Naimiy, H. M.: 1978, Astrophys. Space Sci. 53, 181.

Andersen, J.: 1976, Astron. Astrophys. 47, 467.

Atkins, H. L.: 1971, M.S. Thesis, University of Georgia.

Beltrami, G. and Galeotti, P.: 1970, Mem. Soc. Astron. Ital. 41, 167.

Bolokadze, R. D.: 1953, Perem. Zvezdy 9, 379.

Budding, E. and Najim, N. N.: 1980, Astrophys. Space Sci. 72, 369.

Cester, B., Fedel, B., Giuricin, G., Mardirossian, F., and Mazzetti, M.: 1978, Astron. Astrophys. Suppl. 33, 91.

Chambliss, C. R.: 1983, Astrophys. Space Sci. 89, 15.

Chambliss, C. R. and Leung, K. C.: 1982, Astrophys. J. Suppl. 49, 531.

Daniel, Z.: 1916, Publ. Allegheny Obs. 3, 179.

Duerbeck, H. W.: 1975, Astron. Astrophys. Suppl. 22, 19.

Duerbeck, H. W.: 1976, Astron. Astrophys. 47, 471.

Eaton, J. A.: 1975, Astrophys. J. 197, 379.

Giuricin, G. Mardirossian, F., Mazzetti, M., and Chambliss, C. R.: 1983, Astron. Astrophys. Suppl. 51, 111.

Grygar, J., Cooper, M. L., and Jurkevich, I.: 1972, Bull. Astron. Inst. Czech. $23,147$.

Huffer, C. M. and Kopal, Z.: 1951, Astrophys. J. 114, 297.

Irwin, J. B.: 1947, Astrophys. J. 106, 380.

Klinglesmith, D. A. and Sobieski, S.: 1970, Astron. J. 75, 175.

Koch, R. H., Sobieski, S., and Wood, F. B.: 1963, Publ. Univ. Penna. Astron. Ser. 9, 32. 
Kopal, Z. and Shapley, M. B.: 1956, Jodrell Bank Ann. 1, 141.

Russell, H. N. and Merrill, J. E.: 1952, Contr. Princeton Univ. Obs., No. 26.

Wilson, R. E. and Devinney, E. J.: 1971, Astrophys. J. 166, 304.

Wood, F. B.: 1946, Contr. Princeton Univ. Obs. 21, 25.

Wood, D. B.: 1971, Astron. J. 76, 701.

Wood, D. B.: 1972, 'A Computer Program for Modeling Non-Spherical Eclipsing Binary Star Systems', NASA Publ. X 11072473. 